\title{
Plantas medicinais ajudam a combater a insônia
}

\author{
Medicinal plants help fight insomnia \\ Las plantas medicinales ayudan a combatir el insomnio
}

Caio Franklin Vieira de Figueiredo

ORCID: https://orcid.org/0000-0003-0364-164X Universidade Federal de Campina Grande, Brasil E-mail: caiovieirafigueiredo@gmail.com

Tiago da Nóbrega Albuquerque ORCID: https://orcid.org/0000-0001-8046-8727 Universidade Federal de Campina Grande, Brasil E-mail: tiagofernandes_pb@hotmail.com

Karla da Nóbrega Gomes ORCID: https://orcid.org/0000-0001-9430-4176 Universidade Federal de Campina Grande, Brasil E-mail: karlindagomes@hotmail.com

Sandra Maijane Soares de Belchior ORCID: https://orcid.org/0000-0001-5807-2259 Universidade Federal de Campina Grande, Brasil E-mail: sandrabelchior@hotmail.com

Osvaldo Soares da Silva ORCID: https://orcid.org/0000-0002-4608-0638 Universidade Federal de Campina Grande, Brasil E-mail: osvaldo@ccta.ufcg.edu.br

\begin{abstract}
Resumo
O uso de plantas medicinais faz parte da história da humanidade a muitas décadas, no entanto, essa cultura até então que estava um pouco esquecida vem ganhando espaço novamente. Objetivou-se com esse estudo realizar um levantamento bibliográfico no ano de 2021 de estudos relacionados a meta-analises da insônia. Para os termos descritores, citam-se: Insomnia, herbal medicine and meta-analysis, com ênfase nas revisões voltadas para terapia da insônia. Foram encontrados 34 artigos de revisão, dois foram eliminados após leitura de resumo e 31 não falavam diretamente sobre insônia e sua cura com plantas medicinais e apenas um fazia um levantamento da cura da insônia com plantas através da aromoterapia. Com o auxílio da técnica de meta analise no estudo utilizado observaram que a aromaterapia é eficiente no tratamento da insônia. Fizeram um levantamento de 417 artigos, onde ao final para a pesquisa usaram as respostas de 19 artigos de 2014 a 2020. A insônia é prevalente na população brasileira e o uso de plantas medicinais, com atividade sedativa, destaca-se entre os tratamentos.
\end{abstract}

Palavras-chave: Aromaterapia; Meta análise; Óleos essenciais.

\begin{abstract}
The use of medicinal plants has been part of human history for many decades, however, this culture, which was somewhat forgotten until then, has been gaining ground again. -analyses of insomnia. For the descriptor terms, the following are cited: Insomnia, herbal medicine and meta-analysis, with an emphasis on reviews aimed at insomnia therapy. Thirty-four review articles were found, two were eliminated after reading the abstract and 31 did not speak directly about insomnia and its cure with medicinal plants and only one surveyed the cure for insomnia with plants through aromatherapy. With the help of the meta-analysis technique in the study used, they observed that aromatherapy is efficient in the treatment of insomnia. They carried out a survey of 417 articles, where at the end of the research they used the responses of 19 articles from 2014 to 2020. Insomnia is prevalent in the Brazilian population and the use of medicinal plants, with sedative activity, stands out among the treatments.
\end{abstract}

Keywords: Aromatherapy; Meta analysis; Essencial oils.

\section{Resumen}

El uso de plantas medicinales ha sido parte de la historia de la humanidad desde hace muchas décadas, sin embargo, esta cultura, algo olvidada hasta entonces, ha ido ganando terreno nuevamente.- Análisis del insomnio. Para los términos del descriptor, se citan los siguientes: Insomnio, fitoterapia y metanálisis, con énfasis en las revisiones dirigidas a la terapia del insomnio. Se encontraron treinta y cuatro artículos de revisión, dos fueron eliminados luego de la lectura del resumen y 31 no hablaban directamente sobre el insomnio y su cura con plantas medicinales y solo uno relevó la cura del insomnio con plantas a través de la aromaterapia. Con la ayuda de la técnica de metanálisis utilizada en el estudio, observaron que la aromaterapia es eficaz en el tratamiento del insomnio. Realizaron una 
encuesta de 417 artículos, donde al final de la investigación utilizaron las respuestas de 19 artículos de 2014 a 2020. El insomnio es prevalente en la población brasileña y el uso de plantas medicinales, con actividad sedante, se destaca entre los tratos.

Palabras clave: Aromaterapia; Metaanálisis; Aceites esenciales.

\section{Introdução}

A utilização de plantas com propriedades medicinais faz parte da história da humanidade a muitas décadas, no entanto, essa cultura até então que estava um pouco esquecida vem ganhando espaço novamente (Bruning et al., 2012). Sendo usadas como meios alternativos para tratamento de uma grande quantidade de enfermidades e em alguns casos essa forma de cura é uma das poucas soluções disponíveis para algumas comunidades, quando comparadas aos medicamentos alopáticos. Além da existência de grandes números de espécies vegetais com propriedades terapêuticas o que vem a facilitar sua disponibilidade (Bortoluzzi et al., 2019).

Os princípios ativos presentes em algumas plantas fazem com que elas possam auxiliar no tratamento ou na cura de doenças. Quando a planta apresenta compostos que tem ação farmacológica, é dada a ela a classificação de planta medicinal (Santos \& Trindade, 2017). A insônia é um sentimento subjetivo quando insatisfeito com a qualidade ou quantidade do sono. Este é um sintoma que inclui dificuldade em dar início ou manter o sono, acordar cedo e não conseguir adormecer novamente, sendo o descanso insuficiente para preservar uma boa qualidade de vida, podendo prejudicar causar danos físicos e mentalmente ao indivíduo, e consequentemente gerando prejuízos durante as atividades diárias (Rogério \& Ribeiro, 2021).

Entre 33\% a 50\% da população aflige-se de insônia em alguma parte de sua vida (Prado et al., 2012). Fazendo parte da vida das pessoas e de forma essencial, o sono quando perturbado pode ocasionar diversos danos à saúde, tais como, redução da qualidade de vida, fadiga e prejudicar a função diurna (Khadivzadeh et al., 2018). Esses sintomas podem vim até afetar a concentração e a memória, ocasionar aumento no risco de hipertensão, Alzheimer, doença coronariana, câncer, intenção suicida, ansiedade, obesidade e diabetes mellitus (Prado et al., 2012; Feizi et al., 2018). Entre o grande número de aplicações das plantas medicinais no auxílio de enfermidades, algumas plantas como erva cidreira, valeriana e maracujá tem propriedades que pode ajudar no combate a insônia, sendo geralmente utilizadas através de chás (Santos et al., 2021).

De acordo com Rogério \& Ribeiro (2021) em seu estudo foram analisados os nomes populares, nomes científicos, indicações e riscos em diferentes plantas com propriedades medicinais utilizadas no tratamento da insônia no Brasil. Senda as plantas utilizadas com fins terapêuticos citadas pela maioria número de autores foram: erva cidreira, maracujá, valeriana, lúpulo e lavanda. A camomila destacou-se em relação a sua função da segurança e eficácia, pois não apresentou uma toxicidade relevante. Porém, o uso do hipérico, kava-kava e valeriana devem ser utilizadas com mais cautela e prescritas e vendidas sob orientação médica, devido aos relatos de reações adversas, contraindicações e interações medicamentosas.

Com base na qual uma grande porção da população sofre com problemas relacionados a insônia e procurando alternativas que sejam de fácil acesso a uma grande maioria da população, o presente trabalho teve como objetivo uma pesquisa bibliográfica sobre plantas medicinais que ajudem a reduzir a insônia.

\section{Metodologia}

O presente artigo compreendeu uma revisão bibliográfica cujo objeto foi o tratamento da insônia através de plantas medicinais. Para tanto, foi realizado um levantamento de artigos de revisão sobre a temática compreendendo publicações internacionais no ano de 2021.

Foi realizada uma seleção de conteúdo ancorada na realização de fichamentos capazes de contemplar os principais pontos investigados, tais como: Histórico da utilização de plantas medicinais; Caracterização do número de espécies vegetais com propriedades terapêuticas; Definição de insônia, bem como estatísticas e implicações da mesma na vida dos indivíduos; 
Entre outros aspectos, de modo que o presente trabalho teve como objetivo uma pesquisa bibliográfica sobre plantas medicinais que ajudem a reduzir ou combater esse mal denominado insônia.

Esse tipo de investigação científica de abordagem descritiva e qualitativa foi desenvolvida em etapas que compreenderam a definição do título e objeto; Elaboração dos objetivos; Fundamentação pautada na justificativa de abordagem do tema e problemática, bem como a utilização de critérios para a inclusão ou não de determinados artigos ou fontes, tendo sido priorizados artigos atualizados, de plataformas acadêmicas e disponibilidade on line (Scielo, Biblioteca Virtual de Saúde e Google Acadêmico), em inglês da base de dados Elsevier.

Para os termos descritores, citam-se: Insomnia, herbal medicine and meta-analysis, com ênfase nas revisões voltadas para terapia da insônia. Foram encontrados 34 artigos de revisão, dois foram eliminados após leitura de resumo e 31 não falavam diretamente sobre insônia e sua cura com plantas medicinais e apenas um fazia um levantamento da cura da insônia com plantas através da aromoterapia (Figura 1).

Figura 1. Fluxograma da revisão.

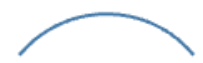

34 artigos de revisão

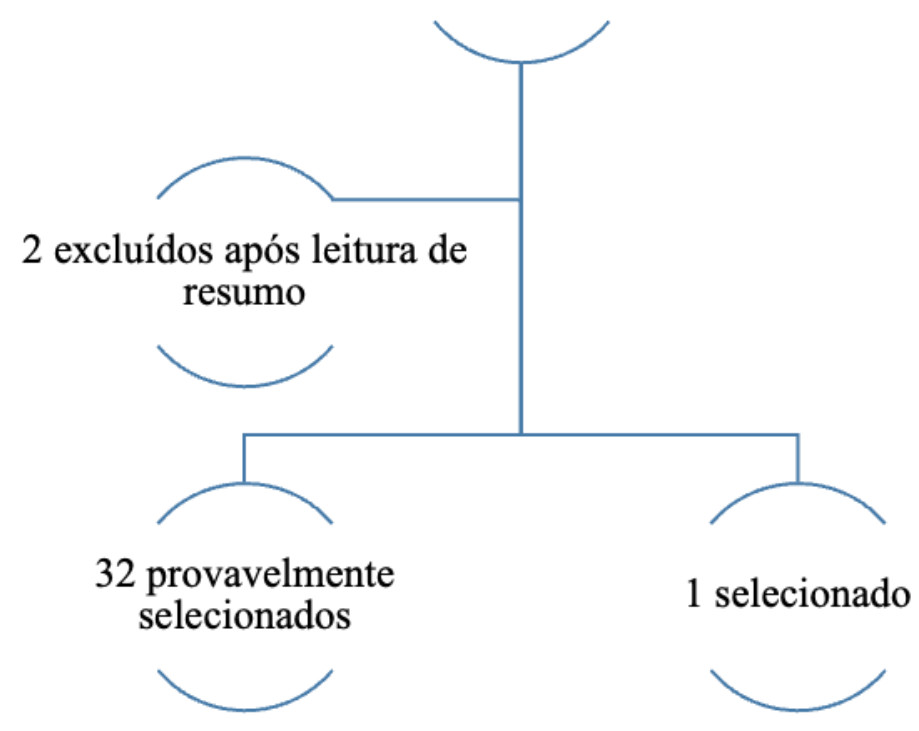

Fonte: Autores.

No Quadro 1 segue o artigo selecionado como base para a pesquisa:

Quadro1. Estudo selecionado.

\begin{tabular}{|c|c|c|}
\hline Autor & Título & Palavras-chave \\
\hline $\begin{array}{c}\text { Tang, Y., Gong, M., Qin, } \\
\text { X., Su, H., Wang, Z., \& } \\
\text { Dong, H. }\end{array}$ & The Therapeutic Effect of & Aromatherapy, Insomnia, Meta-analysis, \\
& Ratherapy on Insomnia: a \\
& Meta-Analysis. & \\
\end{tabular}

Fonte: Autores. 


\section{Resultados e Discussão}

Com o auxílio da técnica de meta analise Tang et al. (2021) observaram que a aromaterapia é eficiente no tratamento da insônia. Fizeram um levantamento de 417 artigos, onde ao final para a pesquisa usaram as respostas de 19 artigos de 2014 a 2020 .

A Aromaterapia é a ciência que estuda o tratamento de doenças, por exemplo a insônia, com o uso de aroma das plantas por meio dos seus óleos essenciais (Ulrich, 2004). Os óleos essenciais são substâncias complexas, voláteis e de fragrância variável, oriundos de qualquer parte da planta, sendo produzidos principalmente pelas famílias Lauraceae, Myrtaceae, Labiateae, Rutaceae, Umbeliferaceae, entre outras, e resultantes do metabolismo secundário das plantas aromáticas (Bandoni, Czepak, 2008). A valeriana e a camomila, por exemplo são bem utilizadas na aromaterapia na forma de óleo para melhorar a qualidade do sono. A erva cidreira foi descoberta e tornou-se a queridinha na aromaterapia.

Por conta de suas propriedades relaxantes e sedativas, descobertas e utilizadas pelos árabes desde o século X para reduzir a ansiedade e o estresse, a erva cidreira e os óleos essenciais derivados da planta ganham cada vez mais espaço na área da aromaterapia. Os efeitos calmante e soporífero, ambos comprovados cientificamente, são os que fazem com que os aromaterapeutas indiquem o uso do óleo essencial tanto para situações comuns de estresse cotidiano, quanto para o tratamento de doenças como a ansiedade, depressão e insônia, definidos como os principais transtornos mentais do século XXI.

Pela dificuldade de encontrar a planta em algumas regiões seus óleos essenciais são muito vendidos. Tang et al. (2021) observaram em sua pesquisa que os autores Afshar et al. (2015), Yu et al. (2015), Ozkaraman et al. (2018), Cheraghbeig ey al. (2019) e Rafii et al. (2020) em suas pesquisas trataram pessoas que tinham insônia devido a problemas de saúde como: cancer, cardiovasculares, depressão etc, com o oleo essencial de lavanda e tiveram bons resultados. A lavanda por possuir folhas aromáticas é utilizada na aromaterapia, com efeitos sedativos, antidepressivos, antiespasmódicos, antibacterianos e anestésicos locais (Adamuchio, 2017). Como também em outras pesquisas utilizadas por Tang et al. (2021) observou-se que utilizaram óleos de amêndoas e de rosas para combater a insônia como aromaterapia.

\section{Conclusão}

O uso de plantas para os tratamentos de distúrbios, tais como a insônia, podem ser tratados ou reduzidos os sintomas através de plantas que em sua constituição apresente compostos que combatem esse mal. Além das plantas serem de fácil acesso para a grande maioria da população, o que faz o uso delas uma boa alternativa no combate ou tratamento da insônia.

A insônia é prevalente na população brasileira e o uso de plantas medicinais, com atividade sedativa, destaca-se entre os tratamentos. Sendo necessário mais estudos para entendermos melhor os princípios ativos das plantas medicinais na cura de algumas doenças ou até mesmo comprovar cientificamente o saber popular.

\section{Referências}

Ballesio, A., Bacaro, V., Vacca, M., Chirico, A., Lucidi, F., Riemann, D., ... \& Lombardo, C. (2021). Does cognitive behaviour therapy for insomnia reduce repetitive negative thinking and sleep-related worry beliefs? A systematic review and meta-analysis. Sleep Medicine Reviews, 55, 101378.

Bortoluzzi, M. M; Schmitt, V; Mazur, C. E. (2020). Efeito fitoterápico de plantas medicinais sobre a ansiedade: uma breve revisão. Research, Society and Development, 9(1), e02911504.

Cénat, J. M., Blais-Rochette, C., Kokou-Kpolou, C. K., Noorishad, P. G., Mukunzi, J. N., McIntee, S. E., ... \& Labelle, P. R. (2021). Prevalence of symptoms of depression, anxiety, insomnia, posttraumatic stress disorder, and psychological distress among populations affected by the COVID-19 pandemic: A systematic review and meta-analysis. Psychiatry research, 295, 113599.

Curtis, F., Laparidou, D., Bridle, C., Law, G. R., Durrant, S., Rodriguez, A., ... \& Siriwardena, A. N. (2021). Effects of cognitive behavioural therapy on insomnia in adults with tinnitus: Systematic review and meta-analysis of randomised controlled trials. Sleep medicine reviews, 56, 101405.

Gardani, M., Bradford, D. R., Russell, K., Allan, S., Beattie, L., Ellis, J., \& Akram, U. (2021). A systematic review and meta-analysis of poor sleep, insomnia symptoms and stress in undergraduate students. Sleep medicine reviews, 101565. 
Hu, N., Wang, C., Liao, Y., Dai, Q., \& Cao, S. (2021). Smoking and Incidence of Insomnia: a systematic review and meta-analysis of cohort studies. Public Health, 198, 324-331.

Ji, X., Ivers, H., Beaulieu-Bonneau, S., \& Morin, C. M. (2021). Complementary and alternative treatments for insomnia/insomnia-depression-anxiety symptom cluster: Meta-analysis of English and Chinese literature. Sleep Medicine Reviews, 101445.

Jing, R., \& Feng, K. (2021). Efficacy of intradermal acupuncture for insomnia: a meta-analysis. Sleep Medicine, 85, 66-74.

Johnson, K. A., Gordon, C. J., Chapman, J. L., Hoyos, C. M., Marshall, N. S., Miller, C. B., \& Grunstein, R. R. (2021). The association of insomnia disorder characterised by objective short sleep duration with hypertension, diabetes and body mass index: A systematic review and meta-analysis. Sleep Medicine Reviews, 101456

Khadvzadeh, T. et al. (2018). A Systematic Reviewand Meta-analysis on the Effect of Herba Medicine to Manage Sleep Dysfunction in Peri and Postmeno pause. Journal of Menopausal Medicine. v. 24, p.92-99.

Li, L., Gan, Y., Zhou, X., Jiang, H., Zhao, Y., Tian, Q., ... \& Lu, Z. (2021). Insomnia and the risk of hypertension: A meta-analysis of prospective cohort studies. Sleep medicine reviews, 56, 101403.

Li, Y., Chen, B., Hong, Z., Sun, Q., Dai, Y., Basta, M., ... \& Qin, Q. (2021). Insomnia symptoms during the early and late stages of the COVID-19 pandemic in China: a systematic review and meta-analysis. Sleep medicine.

Liu, C., Pan, W., Li, L., Li, B., Ren, Y., \& Ma, X. (2021). Prevalence of depression, anxiety, and insomnia symptoms among patients with COVID-19: A meta-analysis of quality effects model. Journal of Psychosomatic Research, 110516.

Ma, Y., Hall, D. L., Ngo, L. H., Liu, Q., Bain, P. A., \& Yeh, G. Y. (2021). Efficacy of cognitive behavioral therapy for insomnia in breast cancer: a metaanalysis. Sleep Medicine Reviews, 55, 101376.

Madrid-Valero, J. J., Rubio-Aparicio, M., Gregory, A. M., Sánchez-Meca, J., \& Ordoñana, J. R. (2021). The heritability of insomnia: Systematic review and meta-analysis of twin studies. Sleep Medicine Reviews, 101437.

Mahmud, S., Hossain, S., Muyeed, A., Islam, M. M., \& Mohsin, M. (2021). The global prevalence of depression, anxiety, stress, and, insomnia and its changes among health professionals during COVID-19 pandemic: A rapid systematic review and meta-analysis. Heliyon, 7(7), e07393.

Maiolino, G., Bisogni, V., Soranna, D., Pengo, M. F., Pucci, G., Vettor, R., ... \& Silvani, A. (2021). Effects of Insomnia and Restless Legs Syndrome on Sleep Arterial Blood Pressure: A Systematic Review and Meta-Analysis. Sleep Medicine Reviews, 101497.

Manzar, M. D., Jahrami, H. A., \& Bahammam, A. S. (2021). Structural validity of the Insomnia Severity Index: A systematic review and meta-analysis. Sleep medicine reviews, 60,101531 .

Maurer, L. F., Schneider, J., Miller, C. B., Espie, C. A., \& Kyle, S. D. (2021). The clinical effects of sleep restriction therapy for insomnia: A meta-analysis of randomised controlled trials. Sleep Medicine Reviews, 101493

Montgomery, M., Baylan, S., \& Gardani, M. (2021). Prevalence of insomnia and insomnia symptoms following mild-Traumatic Brain Injury: a systematic review and meta-analysis. Sleep Medicine Reviews, 101563

Najafpour, Z., Fatemi, A., Goudarzi, Z., Goudarzi, R., Shayanfard, K., \& Noorizadeh, F. (2021). Cost-effectiveness of neuroimaging technologies in management of psychiatric and insomnia disorders: A meta-analysis and prospective cost analysis. Journal of Neuroradiology, 48(5), 348-358.

Pappa, S., Ntella, V., Giannakas, T., Giannakoulis, V. G., Papoutsi, E., \& Katsaounou, P. (2021). Corrigendum to "Prevalence of depression, anxiety, and insomnia among healthcare workers during the COVID-19 pandemic: a systematic review and meta-analysis”[Brain Behav. Immun. 88 (2020) 901-907]. Brain, Behavior, and Immunity, 92, 245

Parsons, C. E., Zachariae, R., Landberger, C., \& Young, K. S. (2021). How does cognitive behavioural therapy for insomnia work? A systematic review and meta-analysis of mediators of change. Clinical Psychology Review, 102027.

Prado, G.F., Carvalho, L.B.C.; Prado, L.B.F. (2012). Insomnia. Brasil: Revista Brasileira de Medicina

Rogério L V F; Ribeiro J C. (2021). Uso de plantas medicinais e medicamentos fitoterápicos em insônia: uma revisão bibliográfica. Brazilian Journal of Health and Pharmacy, . 3(2), 35-44

Santos, R. S; Silva, S. S; Vasconcelos, T. C. L. (2021). Aplicação de plantas medicinais no tratamento da ansiedade: uma revisão da literatura. Brazilian Journal of Development, 7(5), 52060-52074

Santos, V,. Trindade, L. (2017) A enfermagem no uso das plantas medicinais e da fitoterapia com ênfase na saúde pública. Revista cientifica.

Selvanathan, J., Pham, C., Nagappa, M., Peng, P. W., Englesakis, M., Espie, C. A., ... \& Chung, F. (2021). Cognitive Behavioral Therapy for Insomnia in Patients with Chronic Pain-A Systematic Review and Meta-Analysis of Randomized Controlled Trials. Sleep Medicine Reviews, 101460.

Silva, F. C. T., \& Neto, M. L. R. (2021). Psychiatric symptomatology associated with depression, anxiety, distress, and insomnia in health professionals working in patients affected by COVID-19: a systematic review with meta-analysis. Progress in Neuro-Psychopharmacology and Biological Psychiatry, 104, 110057.

Sumsuzzman, D. M., Choi, J., Jin, Y., \& Hong, Y. (2021). Neurocognitive effects of melatonin treatment in healthy adults and individuals with Alzheimer's disease and insomnia: a systematic review and meta-analysis of randomized controlled trials. Neuroscience \& Biobehavioral Reviews. 
Research, Society and Development, v. 11, n. 2, e14611225108, 2022

(CC BY 4.0) | ISSN 2525-3409 | DOI: http://dx.doi.org/10.33448/rsd-v11i2.25108

Sun, N., He, Y., Wang, Z., Zou, W., \& Liu, X. (2021). The effect of repetitive transcranial magnetic stimulation for insomnia: a systematic review and metaanalysis. Sleep Medicine, 77, 226-237.

Tang, Y., Gong, M., Qin, X., Su, H., Wang, Z., \& Dong, H. (2021). The Therapeutic Effect of Aromatherapy on Insomnia: a Meta-Analysis. Journal of Affective Disorders.

Xiang, T., Cai, Y., Hong, Z., \& Pan, J. (2021). Efficacy and safety of Zolpidem in the treatment of insomnia disorder for one month: a meta-analysis of a randomized controlled trial. Sleep Medicine, 87, 250-256.

Xue, T., Wu, X., Chen, S., Yang, Y., Yan, Z., Song, Z., ... \& Wang, Z. (2021). The efficacy and safety of dual orexin receptor antagonists in primary insomnia: a systematic review and network meta-analysis. Sleep medicine reviews, 101573.

Yang, J. (2021). Acupuncture treatment for post-stroke insomnia: A systematic review and meta-analysis of randomized controlled trials. Complementary Therapies in Clinical Practice, 101396.

Zhang, Y., Jiang, X., Liu, J., Lang, Y., \& Liu, Y. (2021). The association between insomnia and the risk of metabolic syndrome: A systematic review and meta-analysis. Journal of Clinical Neuroscience.

Zhao, F. Y., Fu, Q. Q., Kennedy, G. A., Conduit, R., Zhang, W. J., Wu, W. Z., \& Zheng, Z. (2021). Can acupuncture improve objective sleep indices in patients with primary insomnia? A systematic review and meta-analysis. Sleep medicine.

Zhao, W., Van Someren, E. J., Li, C., Chen, X., Gui, W., Tian, Y., ... \& Lei, X. (2021). EEG spectral analysis in insomnia disorder: A systematic review and meta-analysis. Sleep Medicine Reviews, 101457. 